\title{
Low FT3 is an independent marker of disease severity in patients hospitalized for COVID-19
}

\author{
Aditya Dutta', Ganesh Jevalikar', Rutuja Sharma', Khalid J Farooqui', Shama Mahendru', Arun Dewan², \\ Sandeep Bhudiraja ${ }^{2}$ and Ambrish Mithal ${ }^{1}$
}

${ }^{1}$ Institute of Endocrinology and Diabetes, Max Healthcare, Saket, New Delhi, India

${ }^{2}$ Institute of Internal Medicine, Max Healthcare, Saket, New Delhi, India

\begin{abstract}
Aim: To study the prevalence of thyroid dysfunction and its association with disease severity in hospitalized patients of coronavirus disease-19 (COVID-19).

Methods: In this retrospective cohort study, thyroid function tests (TFT) of 236

hospitalized patients of COVID-19 along with demographic, comorbid, clinical,

biochemical and disease severity records were analysed. Patients were divided into

previous euthyroid or hypothyroid status to observe the effect of prior hypothyroidism

on the severity of COVID-19.

Results: TFT abnormalities were common. Low free T3 (FT3), high thyroid-stimulating hormone (TSH) and low TSH were seen in 56 (23.7\%), 15 (6.4\%) and 9 (3.8\%) patients, respectively. The median levels of TSH ( $2.06 \mathrm{vs} 1.26 \mathrm{mIU} / \mathrm{mL}, P=0.001)$ and FT3 (2.94 vs $2.47 \mathrm{pg} / \mathrm{mL}, P<0.001$ ) were significantly lower in severe disease. Previous hypothyroid status $(n=43)$ was associated with older age, higher frequency of comorbidities, higher FT4 and lower FT3. TFT did not correlate with markers of inflammation (except lactate dehydrogenase); however, FT3 and TSH negatively correlated with outcome severity score and duration of hospital stay. Cox regression analysis showed that low FT3 was associated with severe COVID-19 $(P=0.032, \mathrm{HR} 0.302 ; \mathrm{Cl} 0.101-0.904)$, irrespective of prior hypothyroidism.

Conclusions: Functional thyroid abnormalities (low FT3 and low TSH) are frequently seen in hospitalized patients of COVID-19. Although these abnormalities did not correlate with markers of inflammation, this study shows that low FT3 at admission independently predicts the severity of COVID-19.
\end{abstract}

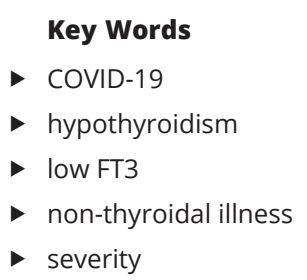

Endocrine Connections (2021) 10, 1455-1462

\section{Introduction}

Severe acute respiratory syndrome coronavirus 2 (SARS-CoV-2)-induced coronavirus disease-19 (COVID-19) pandemic continues to affect the world. Although SARS-CoV-2 results primarily in an acute respiratory illness, its direct and indirect effects (due to pro-inflammatory cytokine storm) on several endocrine organs and their axes have been elucidated $(1,2,3)$. Hypothalamicpituitary-thyroid (HPT) axis is an essential endocrine axis that regulates energy expenditure and homeostasis.
Recent studies, reviews and epidemiological data have shed light on the involvement of HPT axis and thyroid gland in COVID-19 $(1,2,3,4,5,6,7,8,9,10,11)$. The changes in HPT axis associated with COVID-19 include impaired thyroid-stimulating hormone (TSH) secretion, decreased T3 and T4 (non-thyroidal illness; NTI), decreased activity of deiodinases and thyrotoxicosis (10). The pathophysiology behind these changes has been explained by the cytokine storm associated with COVID-19 (low TSH, NTI) and direct

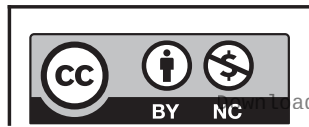

This work is licensed under a Creative Commons Attribution-NonCommercial 4.0 International License. ded from Bioscientifica.com at 04/26/2023 03:33:15AM 
thyroid-follicular apoptosis causing destructive thyroiditis (thyrotoxicosis). SARS-CoV-2 may gain entrance to the endocrine organs (including thyroid gland) using angiotensin-converting enzyme 2 (ACE2) receptor, thereby directly afflicting the endocrine system (12).

Hypothyroidism is a common comorbidity seen in patients hospitalized with COVID-19 (13). Prior history of thyroid disorders and the risk of COVID-19 severity and progression is a contentious topic. While a clinical study and another meta-analysis have demonstrated enhanced risk of severe infection $(14,15)$, two large cohort studies have ruled out worse prognosis of COVID-19 in patients with pre-existing thyroid disorders $(16,17)$.

Despite the knowledge gained over the past year, there is dearth of data showing a clinical association of detrimental effects of thyroid-axis dysfunction and previous hypothyroid status on the inflammatory markers, disease severity and outcomes in patients hospitalized for COVID-19. In this retrospective single-centre study, we compiled the baseline parameters including thyroid function tests (TFT) and comorbidities (including previous hypothyroid status) in consecutive hospitalized Indian patients and studied their association with inflammatory markers, severity and mortality of COVID-19.

\section{Materials and methods}

\section{Study design}

This is a retrospective, single-centre, cohort study carried out at a tertiary care, designated as COVID-19 treatment centre, situated in New Delhi, India. Hospitalized patients were enrolled from July 9, 2020, to August 8, 2020, and were observed till the time of discharge or death while in the hospital. The study was approved by the Max Healthcare Ethics Committee, New Delhi, India. A waiver of consent was sought because retrospective patient data were used, and the study protocol did not affect the treatment protocol or patient identity in any way.

\section{Participants}

The data of this study are derived from our previously published prospective cohort of 420 hospitalized patients of COVID-19 (18). A total of 236 patients whose TFT were available were included. These included consecutive patients hospitalized with COVID-19 infection proven positive by nasal and/or nasopharyngeal swab for SARS-CoV-2 by RT-PCR method. Asymptomatic patients were not hospitalized, except in 13 cases where the patient was either a healthcare worker or home isolation was not possible.

\section{Measurements}

Clinical data were collected from the electronic medical records including age, sex, presence of comorbidities, presenting symptoms, duration of symptoms, anthropometry, blood pressure, baseline oxygen saturation $\left(\mathrm{SpO}_{2}\right)$, results of laboratory evaluation and treatment received. All patients were assigned a severity score based on the World Health Organization (WHO) ordinal scale for clinical improvement (OSCI) at hospital admission (baseline) and the highest score during the hospital stay (outcome). Based on the outcome OSCI scores, patients were classified as hospitalized mild disease (3, no oxygen therapy and 4, oxygen by mask or nasal prongs) and hospitalized severe disease $(5$, non-invasive ventilation or high-flow oxygen; 6, intubation and mechanical ventilation; 7, ventilation plus other organ support like inotropes/renal replacement therapy (RRT)/extracorporeal membrane oxygenation; and 8, death). All patients had a blood sampling done at admission to determine TSH (normal: 0.35-5.6 mIU/mL), free T4 (FT4; normal: 0.58-1.64 $\mathrm{ng} / \mathrm{dL}$ ) and free T3 (FT3; normal: 2.6-4.8 pg/mL) in addition to the standard COVID-19 protocol which included assessment of inflammatory markers, C-reactive protein (CRP), interleukin-6 (IL-6), D-dimer, ferritin and lactate dehydrogenase (LDH). The levels of TSH, FT4 and FT3 were determined using chemiluminescence immunoassay (Beckman Coulter Unicel DxI 800 system). TFT was performed only once at baseline.

\section{Objectives}

There were two primary objectives. First is to evaluate hospitalized patients of COVID-19 for the presence and prevalence of thyroid dysfunction, and second is to assess the association of baseline TFT with severity and mortality outcome of COVID-19. Secondary objectives were to identify differences in clinical, biochemical and outcome parameters of patients with previous hypothyroid and euthyroid status; to study the correlation between TFT and markers of inflammation, disease severity and the duration of hospital stay; and to establish the effect of previous hypothyroid status on disease severity.

\section{Statistical analysis}

Statistical analysis was performed using IBM SPSS statistics software version 23.0 (IBM Corp). Normality of

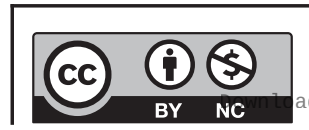

This work is licensed under a Creative Commons Attribution-NonCommercial 4.0 International License. ded from Bioscientifica.com at 04/26/2023 03:33:15AM 
continuous variables was analysed using KolmogorovSmirnov test. Categorical variables were presented as frequency and percentage, whereas continuous variables were presented as median and range. Chisquare test was used to compare differences between categorical variables, and Mann-Whitney $U$ test was used to compare continuous variables. Spearman's rho was used to study the correlation of TFTs with inflammatory markers and outcome severity score. A Cox regression hazard model (using duration of hospital stay as the time-dependent variable) was analysed to study the effect of TFT and other baseline parameters found to be different between hypothyroid and euthyroid individuals on severity of COVID-19. A $P$ value $<0.05$ was considered statistically significant.

\section{Results}

\section{Baseline patient characteristics and overall outcomes of COVID-19 infection}

A total of 236 patients (77 females, 159 males) were included with a median age of 54 years (15-91). Anthropometry was available for 75 patients, and the median BMI was $27.7 \mathrm{~kg} / \mathrm{m}^{2}$ (15.7-46.4). At least one comorbid condition was present in 164 (69.5\%) patients. Comorbid conditions included diabetes $(119,50.4 \%)$, hypertension (102, 43.2\%), hypothyroidism (43, 18.2\%), coronary artery disease (CAD) $(19,8 \%)$, respiratory disease $(15,6.4 \%)$, cancer $(8,3.4 \%)$ and chronic kidney disease (CKD) $(6,2.5 \%)$. The majority of patients $(223,94.5 \%)$ were symptomatic cases with a median symptom duration of 5 days (1-20). At baseline, a total of 222 (94.1\%) patients had mild disease (no oxygen requirement in 181 and lowflow oxygen requirement in 41), whereas 14 (5.9\%) patients had severe disease (high-flow oxygen in 13 and intubation in 1). One hundred fifty (63.6\%) patients received steroids in doses ranging from methylprednisolone (or equivalent) $40 \mathrm{mg}$ once to $60 \mathrm{mg}$ thrice daily. During the hospital stay, 36 (15.3\%) patients had severe outcomes, including mortality in 11 (4.7\%), intubation in $3(1.3 \%)$, and high-flow oxygen/non-invasive ventilation in 22 (9.3\%) patients. A total of $143(60.6 \%)$ patients did not require any supplemental oxygen and $57(24.2 \%)$ required low-flow oxygen. Admission to intensive care unit (ICU), inotropic support, and RRT was required 40 (16.9\%), 13 (5.5\%) and $4(1.7 \%)$ patients each. The median duration of hospital stay was 8 days (1-44). Median duration of ICU stay was 9 days (1-34).

\section{TFT in the study population}

The median TSH, FT4 and FT3 levels were $1.9 \mathrm{mIU} / \mathrm{mL}$ (0.04-39.15), $0.99 \mathrm{ng} / \mathrm{dL}(0.57-1.7)$ and $2.86 \mathrm{pg} / \mathrm{mL}$ (1.81-4.27), respectively. A total of 15 (6.4\%) patients had TSH more than the upper limit of normal; however, only 6 patients had a TSH $>10 \mathrm{mIU} / \mathrm{mL}$. Low TSH was seen in nine (3.8\%) patients. Most patients $(234,99.6 \%)$ were clinically and biochemically euthyroid with FT4 levels in the normal range. FT3 levels were the most variable amongst TFT. Fiftysix (23.7\%) patients had low FT3 suggestive of NTI, which was the most common abnormality. At admission, the median levels of TSH (2.06 vs $1.26 \mathrm{mIU} / \mathrm{mL}, P=0.001)$ and FT3 (2.94 vs $2.47 \mathrm{pg} / \mathrm{mL}, P<0.001$ ) were significantly lower in severe disease as compared to mild disease (Table 1).

\section{Comparison of euthyroid and hypothyroid patients}

Previously, hypothyroid patients were significantly older (63 vs 53 years, $P=0.002$ ), had a greater percentage of comorbidities including hypertension (65.1 vs $38.3 \%$, $P=0.001)$ and CKD (9.3 vs $1 \%, P=0.002)$ and lower median $\mathrm{SpO}_{2}$ (96 vs $97 \%, P=0.006$ ) at presentation than euthyroid patients (Table 2). Other demographic parameters such as sex distribution, duration of symptoms, symptomatic cases and baseline OSCI were similar across the two groups. Except ferritin, which was higher in the hypothyroid patients (206.1 vs $143.5 \mathrm{ng} / \mathrm{mL}, P=0.032$ ), there was no intergroup differences in markers of inflammation (CRP, IL-6, D-dimer and LDH). Baseline TFT differed significantly across the two groups. While FT3 was significantly lower ( 2.67 vs $2.96 \mathrm{pg} / \mathrm{mL}, P=0.006$ ) in the hypothyroid group, FT4 was significantly lower ( 0.97 vs $1.12 \mathrm{ng} / \mathrm{dL}, P=0.017$ ) in the euthyroid group and TSH was similar among the two groups. Except for the increased requirement of ICU (27.9 vs $14.5 \%, P=0.034)$ in the hypothyroid group, there were no differences in clinical outcomes/severity of the two groups with regard to outcome OSCI scores, the proportion of severe cases, mortality, oxygen administration, inotropic support and RRT.

To evaluate the impact of confounding factors (age and comorbidities) on COVID-19, a sub-group analysis (euthyroid/hypothyroid patients; $n=148 / 24$ )

Table 1 Comparison of TFT parameters based on severity.

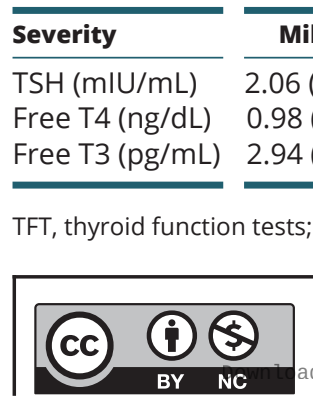

\begin{tabular}{|c|c|c|}
\hline Mild $(n=200)$ & Severe $(n=36)$ & P value \\
\hline $2.06(0.04-39.15)$ & $1.26(0.07-35.49)$ & 0.001 \\
\hline $0.98(0.58-1.7)$ & $1.12(0.57-1.65)$ & 0.08 \\
\hline $2.94(1.73-4.27)$ & $2.47(1.61-3.96)$ & $<0.001$ \\
\hline
\end{tabular}

This work is licensed under a Creative Commons Attribution-NonCommercial 4.0 International License. ed from Bioscientifica.com at 04/26/2023 03:33:15AM via free access 
Table 2 Comparison (baseline and in-hospital stay) of previous euthyroid and previous hypothyroid groups.

\begin{tabular}{|c|}
\hline Characteristics \\
\hline Age (years; $n=236$ ) \\
\hline Female sex \\
\hline $\mathrm{BMI}\left(\mathrm{kg} / \mathrm{m}^{2} ; n=75\right)$ \\
\hline $\mathrm{SBP}(\mathrm{mmHg} ; n=236)$ \\
\hline $\mathrm{DBP}(\mathrm{mmHg} ; n=236)$ \\
\hline Comorbidities $(n=236)$ \\
\hline Diabetes $(n=236)$ \\
\hline Hypertension $(n=236)$ \\
\hline $\mathrm{CAD}(n=236)$ \\
\hline Respiratory $(n=236)$ \\
\hline $\operatorname{CKD}(n=236)$ \\
\hline Cancer $(n=236)$ \\
\hline Others $(n=236)$ \\
\hline Symptomatic cases $(n=236)$ \\
\hline Duration of symptoms (days; $n=215$ ) \\
\hline Baseline $\mathrm{SpO}_{2}(\% ; n=229)$ \\
\hline Baseline OSCI $(n=236)$ \\
\hline 3.0 \\
\hline 4.0 \\
\hline 5.0 \\
\hline 7.0 \\
\hline Hospital stay $(n=236)$ \\
\hline Outcome OSCI $(n=236)$ \\
\hline 3.0 \\
\hline 4.0 \\
\hline 5.0 \\
\hline 6.0 \\
\hline 7.0 \\
\hline 8.0 \\
\hline Change in severity $(n=236)$ \\
\hline Severe cases $(n=236)$ \\
\hline Oxygen $(n=236)$ \\
\hline Oxygen flow $(n=236)$ \\
\hline Low flow \\
\hline High flow \\
\hline ICU admission $(n=236)$ \\
\hline ICU days $(n=40)$ \\
\hline $\mathrm{RRT}(n=236)$ \\
\hline Inotropic support $(n=236)$ \\
\hline Mortality $(n=236)$ \\
\hline $\mathrm{CRP}(\mathrm{mg} / \mathrm{L} ; n=228)$ \\
\hline IL-6 (pg/mL; $n=198)$ \\
\hline $\mathrm{LDH}(\mathrm{U} / \mathrm{L} ; n=224)$ \\
\hline D-dimer $(n g / m L ; n=233)$ \\
\hline Ferritin $(\mathrm{ng} / \mathrm{mL}, n=226)$ \\
\hline Free T4 (ng/dL; $n=235)$ \\
\hline Free T3 $(\mathrm{pg} / \mathrm{mL} ; n=236)$ \\
\hline $\mathrm{TSH}(\mathrm{mIU} / \mathrm{mL} ; n=235)$ \\
\hline
\end{tabular}

\begin{tabular}{c}
\hline Previously euthyroid $(n=193)$ \\
\hline $53(15-89)$ \\
$58(40.6 \%)$ \\
$27.7(15.7-46.4)$ \\
$130(99-178)$ \\
$80(50-100)$ \\
$121(62.7 \%)$ \\
$96(49.7 \%)$ \\
$74(38.3 \%)$ \\
$14(7.3 \%)$ \\
$12(6.2 \%)$ \\
$2(1 \%)$ \\
$7(3.6 \%)$ \\
$36(18.7 \%)$ \\
$183(94.8 \%)$ \\
$5(1-20)$ \\
$97(66-100)$
\end{tabular}

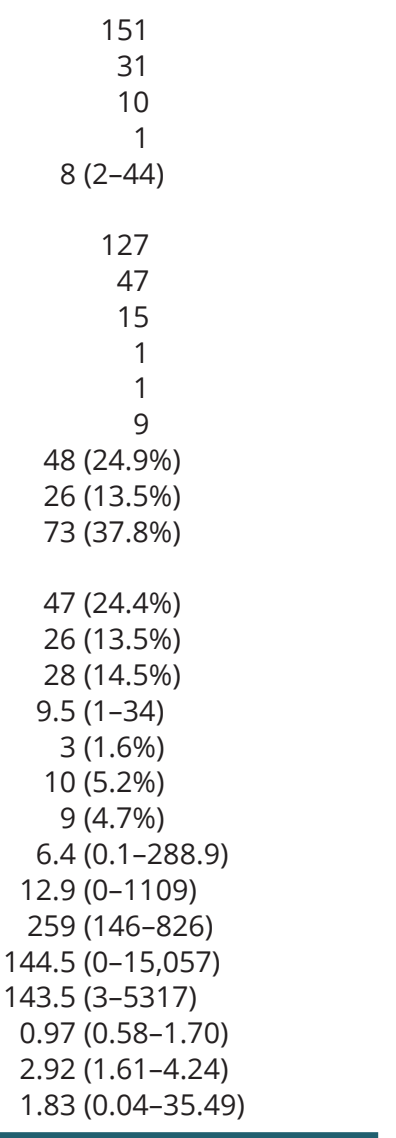

\begin{tabular}{c}
\hline Previously hypothyroid $(n=43)$ \\
\hline $63(31-91)$ \\
$19(44.2 \%)$ \\
$27.8(23.5-35.6)$ \\
$130(105-180)$ \\
$80(60-100)$ \\
$34(79.1 \%)$ \\
$23(46.5 \%)$ \\
$28(65.1 \%)$ \\
$5(11.6 \%)$ \\
$3(6.9 \%)$ \\
$4(9.3 \%)$ \\
$1(2.3 \%)$ \\
$13(30.2 \%)$ \\
$40(93 \%)$ \\
$5(1-15)$ \\
$96(75-99)$
\end{tabular}

\begin{tabular}{c}
\hline P value \\
\hline 0.002 \\
0.074 \\
0.601 \\
0.157 \\
0.795 \\
0.041 \\
0.657 \\
0.001 \\
0.354 \\
0.854 \\
0.002 \\
0.67 \\
0.09 \\
0.641 \\
0.967 \\
0.006
\end{tabular}

30
10
3
0
$10(1-25)$
23
10
7
0
1
2

$13(30.2 \%)$

0.708

$10(23.3 \%)$

$20(46.5 \%)$

0.107

0.292

$10(23.3 \%)$

$9(20.9 \%)$

$12(27.9 \%)$

0.456

$8.5(3-19)$

$1(2.3 \%)$

$3(6.9 \%)$

$2(4.7 \%)$

$9(0.4-254.8)$

$19.7(0-1158)$

$255(129-466)$

$176(0-3888)$

$206.1(16.3-7158.4)$

$1.12(0.57-1.56)$

$2.67(1.64-4.27)$

$2.43(0.15-39.15)$

0.084

0.445

0.034

0.590

0.723

0.641

0.997

0.353

0.061

0.884

0.15

0.032

0.017

0.006

0.143

CAD, coronary artery disease; CKD, chronic kidney disease; CRP, C-reactive protein; DBP, diastolic blood pressure; ICU, intensive care unit; IL-6, interleukin-6; LDH, lactate dehydrogenase; OSCI, ordinal scale for clinical improvement; RRT, renal replacement therapy, SBP, systolic blood pressure; $\mathrm{SpO}_{2}$, baseline oxygen saturation; $\mathrm{TSH}$, thyroid-stimulating hormone.

was performed after excluding 64 elderly patients $(\geq 65$ years). Some of the previous associations remained, while others were lost. Hypothyroid patients had more frequent comorbidities (overall and specifically, respiratory and CKD) than euthyroid patients. However, now the prevalence of hypertension was similar across the subgroups. Age, sex distribution, duration of symptoms, symptomatic cases, baseline severity score and markers of inflammation were similar across the sub-groups. $\mathrm{SpO}_{2}$ at presentation was significantly lower $(P=0.016)$

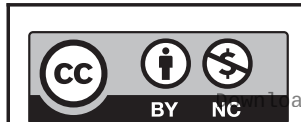

This work is licensed under a Creative Commons Attribution-NonCommercial 4.0 International License. ded from Bioscientifica,com at 04/26/2023 03:33:15AM via free access 
in hypothyroid patients. Although FT3 was lower in hypothyroid patients $(P=0.024)$, the distribution of FT4 and TSH was similar between the sub-groups. There were no differences in the outcome OSCI, mortality, ICU admission, ICU days, oxygen administration, inotropic support and RRT between the two sub-groups. However, hypothyroid patients were likely to have a longer hospital stay $(P=0.05)$.

\section{Correlation of TFT with inflammatory markers and outcome severity scores}

We found a weak negative correlation between FT3 and LDH $(P<0.001$, correlation coefficient (CC): -0.315$)$ and a weak positive correlation between FT4 and $\mathrm{LDH}(P<0.001$, CC: 0.31$)$. No correlation could be found between TFT (TSH and thyroid hormones) and other markers of inflammation (CRP, IL-6, D-dimer and ferritin). Outcome OSCI was found to be positively and negatively correlated with FT4 $(P=0.001, \mathrm{CC}: 0.21)$ and FT3 $(P<0.001, \mathrm{CC}$ : -0.421 ), respectively (Table 3 ). The duration of hospital stay correlated negatively with both FT3 and TSH.

\section{TSH, thyroid hormones and their association with severe COVID-19 and mortality}

FT3 was low in 56 patients in this cohort. On considering FT3 as a categorical variable (low/normal), low FT3 was associated with severe COVID-19 $(P<0.001)$ and mortality $(P<0.001)$ (Table 4). This association could not be shown with FT4 and TSH. A Cox regression analysis was done to ascertain the effect of age, comorbidities (prior hypertension, CKD and hypothyroidism), baseline $\mathrm{SpO}_{2}$, FT4, FT3 on the severity of COVID-19. The regression model was significant $(P=0.003)$. A low FT3 $(P=0.032, \mathrm{HR}$ 0.302; CI 0.101-0.904) and a low baseline $\operatorname{SpO}_{2}(P=0.003$, HR 0.93; CI 0.886-0.976) were found to be associated with severity of COVID-19, irrespective of previous hypothyroid/ comorbid status (Table 5).

\section{Discussion}

In this study comprising 236 hospitalized patients of COVID-19, we found that functional thyroid abnormalities (low FT3 and low TSH) are common at presentation and more prevalent in severe illness. We noted that patients with previous hypothyroid status (irrespective of age) had frequent comorbidities, lower $\mathrm{SpO}_{2}$, lower FT3 at presentation and a longer hospital stay. No association could be found between markers of inflammation (except LDH) and thyroid hormones or TSH. FT3 was found to have inverse correlation with the outcome OSCI. The Cox regression model suggested that FT3 is an independent risk factor for the severity of COVID-19, irrespective of previous hypothyroid status.

The abnormalities of TFT seen in this study included NTI (Low FT3; 23.7\%), HPT axis suppression (Low TSH; $3.8 \%)$ and high TSH (6.4\%). These findings have been well documented previously $(1,2,4,5,6,7,8,10)$. Both FT3 and TSH were lower in patients with severe COVID-19 compared to mild cases. The reasons behind these functional abnormalities are as follows. First, the proinflammatory cytokine storm associated with COVID-19 (or any other critical illness) can result in suppression of pituitary TSH production and 5'-deiodinase activity. Secondly, the use of pharmacological doses of steroids in severe COVID-19 could have had a suppressive effect on pituitary TSH secretion and peripheral conversion of T4 to T3. Thirdly, except one patient, all cases were clinically and biochemically euthyroid (normal FT4). Patients with elevated TSH $(>5.6 \mathrm{mIU} / \mathrm{mL})$ in our study included cases with newly diagnosed subclinical/overt hypothyroidism, previously partially treated hypothyroidism or recovering

Table 3 Correlation of TFT parameters with the markers of inflammation and hospital stay.

\begin{tabular}{|c|c|c|c|c|c|c|}
\hline \multirow[b]{2}{*}{ Parameter } & \multicolumn{2}{|c|}{ FT3 } & \multicolumn{2}{|c|}{ FT4 } & \multicolumn{2}{|c|}{ TSH } \\
\hline & $\mathrm{CC}$ & $P$ value & $\mathrm{CC}$ & $P$ value & $\mathrm{CC}$ & $P$ value \\
\hline $\mathrm{LDH}$ & -0.315 & $<0.001$ & 0.310 & $<0.001$ & -0.163 & 0.015 \\
\hline IL-6 & 0.103 & 0.15 & 0.021 & 0.766 & 0.028 & 0.698 \\
\hline Ferritin & 0.022 & 0.743 & 0.046 & 0.489 & 0.008 & 0.907 \\
\hline CRP & 0.095 & 0.154 & 0.021 & 0.752 & 0.011 & 0.872 \\
\hline D-dimer & 0.036 & 0.586 & 0.083 & 0.21 & -0.026 & 0.693 \\
\hline Outcome OSCI & -0.421 & $<0.001$ & 0.21 & 0.001 & -0.12 & 0.066 \\
\hline Hospital stay & -0.351 & $<0.001$ & 0.063 & 0.336 & -0.138 & 0.035 \\
\hline
\end{tabular}

CRP, C-reactive protein; FT3, free T3; FT4, free T4; IL-6, interleukin-6; LDH, lactate dehydrogenase; OSCI, ordinal scale for clinical improvement; TFT, thyroid function tests. 
Table 4 Abnormal TFT parameters and their association with severity of COVID-19.

\begin{tabular}{|c|c|c|c|}
\hline Parameter & Mild disease & Severe disease & $P$ value \\
\hline Low FT3 $(n=236)$ & $33(16.5 \%)$ & 23 (63.9\%) & $<0.001$ \\
\hline Low TSH $(n=235)$ & $5 / 199(2.5 \%)$ & $4 / 36(11.1 \%)$ & 0.033 \\
\hline High TSH $(n=235)$ & $13(6.5 \%)$ & $2 / 36(5.6 \%)$ & 0.825 \\
\hline
\end{tabular}

FT3, free T3; TFT, thyroid function tests; TSH, thyroid-stimulating hormone.

TSH of NTI. Unlike previous studies $(3,4,7)$, no cases of thyrotoxicosis were seen.

Previous hypothyroid status was associated with older age, higher frequency of comorbidities, lower median $\mathrm{SpO}_{2}$, higher prevalence of ICU admission, higher ferritin, higher FT4 and lower FT3. It may be argued that patients with hypothyroidism are older and may have increased burden of diabetes and cardiovascular comorbidities $(19,20)$. However, even after excluding elderly patients $(n=64)$, previous hypothyroid patients had higher frequency of comorbidities and lower baseline $\mathrm{SpO}_{2}$. Overall, hypothyroid patients had higher FT4 than euthyroid counterparts. This is well explained from the circumstance that hypothyroid patients were on supplemental doses of thyroxine, and it also reaffirms the fact that eventually even FT4 levels decrease in NTI.

No correlation could be established between TFT and markers of inflammation (CRP, IL-6, D-dimer and ferritin), except LDH. The latter correlated positively with FT4 and negatively with FT3 and TSH. Our findings are similar to those reported earlier (5). Previously, studies have also found negative correlation between TSH/FT3 and CRP (2, $5,7,8)$. Although the clinical implications are uncertain, this correlation may represent the bidirectional effect (increased inflammation: LDH/CRP, and decreased conversion: FT4 $\rightarrow$ FT3) of the cytokine storm seen in COVID-19. Both the WHO outcome OSCI and the duration of hospital stay had a negative correlation with FT3 and TSH, a result that resonates with severe COVID-19.
For COVID-19 infection, low FT3 (NTI) has been associated with severe disease in previous studies $(1,2$, $5,6)$. Our results showed low FT3 to be associated with both severity and mortality in COVID-19. Moreover, in the Cox regression analysis, low FT3 was found to be an independent predictor of severity of COVID-19, irrespective of prior hypothyroid status. From this finding, it may be inferred that previous hypothyroid status does not affect the progression/severity and that low FT3 can be considered as a marker of severe disease in COVID-19.

\section{Conclusion}

Functional abnormalities in HPT axis are common in hospitalized patients of COVID-19 with low FT3/NTI being the most frequent. The occurrence of these abnormalities may be attributed to the cytokine storm associated with COVID-19. While low FT3 may not correlate with markers of inflammation, it does predict the disease severity and is associated with mortality in COVID-19. Previous hypothyroid status has no bearing on the prognosis of COVID-19.

\section{Key messages}

\section{Current research questions}

1) What are the types of thyroid function abnormalities in hospitalized patients of COVID-19?

2) Can thyroid function tests done at baseline predict severity of COVID-19?

3) What is the effect of previous hypothyroid status on COVID-19 severity?

4) Is there any association between markers of inflammation and the TFT in hospitalized patients of COVID-19?

Table 5 Results of the Cox regression analysis.

\begin{tabular}{|c|c|c|c|c|c|}
\hline \multirow[b]{2}{*}{ Parameter } & \multirow[b]{2}{*}{ B } & \multirow[b]{2}{*}{ Sig } & \multirow[b]{2}{*}{$\operatorname{Exp}(B)$} & \multicolumn{2}{|c|}{$95 \%$ Cl for $\operatorname{Exp}(B)$} \\
\hline & & & & Lower & Upper \\
\hline FT3 & -1.199 & 0.032 & 0.302 & 0.101 & 0.904 \\
\hline FT4 & 0.297 & 0.671 & 1.346 & 0.341 & 5.039 \\
\hline $\mathrm{SpO}_{2}$ & -0.073 & 0.003 & 0.93 & 0.886 & 0.976 \\
\hline Age & 0.02 & 0.232 & 1.021 & 0.987 & 1.055 \\
\hline Comorbidities & -1.002 & 0.193 & 0.367 & 0.081 & 1.658 \\
\hline Hypothyroidism & 0.208 & 0.612 & 1.231 & 0.552 & 2.749 \\
\hline
\end{tabular}

$\mathrm{FT}$, free T3; FT4, free T4; $\mathrm{SpO}_{2}$, baseline oxygen saturation.

https://ec.bioscientifica.com https://doi.org/10.1530/EC-21-0362 (c) 2021 The authors Published by Bioscientifica Ltd

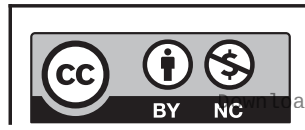

This work is licensed under a Creative Commons Attribution-NonCommercial 4.0 International License. ded from Bioscientifica.com at 04/26/2023 03:33:15AM via free access 


\section{What is already known on the subject?}

1) SARS-CoV-2 may gain entrance to the endocrine organs (including thyroid gland) using ACE2 receptor, thereby directly afflicting the endocrine system.

\section{Main messages}

1) Functional thyroid abnormalities (low FT3 and low TSH) are frequently seen in hospitalized patients of COVID-19.

2) Low FT3 at admission independently predicts severity of COVID-19.

3) Previous hypothyroid status has no bearing on the prognosis of COVID-19.

4) There is no meaningful association between TFT and markers of inflammation.

\section{Declaration of interest}

The authors declare that there is no conflict of interest that could be perceived as prejudicing the impartiality of the research reported.

\section{Funding}

This work did not receive any specific grant from any funding agency in the public, commercial or not-for-profit sector.

\section{Availability of data and material}

Data can be obtained from the corresponding author after appropriate request.

\section{Ethics approval}

Institutional ethics committee approval was obtained.

\section{Author contribution statement}

G J, A M conceived the study, managed the patients, collected the data and edited the manuscript. A D performed interpretation of data and statistical analysis, drafted and edited the manuscript and reviewed the literature. R S, K J F, S M, A D and S B managed the patients, collected the data and supervised patient management.

\section{Acknowledgements}

The authors would like to thank Miss Anshu, Miss Anjali and Mr Bhupendra, diabetes educators of the Institute of Diabetes and Endocrinology, Max Superspeciality Hospital, for their help in data collection and compilation.

\section{References}

1 Chen M, Zhou W \& Xu W. Thyroid function analysis in 50 patients with COVID-19: a retrospective study. Thyroid 202131 8-11. (https:// doi.org/10.1089/thy.2020.0363)
2 Zou R, Wu C, Zhang S, Wang G, Zhang Q, Yu B, Wu Y, Dong H, Wu G, Wu S, et al. Euthyroid sick syndrome in patients with COVID-19. Frontiers in Endocrinology 202011 566439. (https://doi.org/10.3389/ fendo.2020.566439)

3 Lania A, Sandri MT, Cellini M, Mirani M, Lavezzi E \& Mazziotti G. Thyrotoxicosis in patients with COVID-19: the THYRCOV study. European Journal of Endocrinology 2020183 381-387. (https://doi. org/10.1530/EJE-20-0335)

4 Sen K, Sinha A, Sen S, Chakraborty S \& Alam MS. Thyroid function test in COVID-19 patients: a cross-sectional study in a tertiary care hospital. Indian Journal of Endocrinology and Metabolism 202024 532-536. (https://doi.org/10.4103/ijem.IJEM_779_20)

5 Lui DTW, Lee CH, Chow WS, Lee ACH, Tam AR, Fong CHY, Law CY, Leung EKH, To KKW, Tan KCB, et al. Thyroid dysfunction in relation to immune profile, disease status, and outcome in 191 patients with COVID-19. Journal of Clinical Endocrinology and Metabolism 2021106 e926-e935. (https://doi.org/10.1210/clinem/dgaa813)

6 Malik J, Malik A, Javaid M, Zahid T, Ishaq U \& Shoaib M. Thyroid function analysis in COVID-19: a retrospective study from a single center. PLoS ONE 202116 e0249421. (https://doi.org/10.1371/journal. pone.0249421)

7 Khoo B, Tan T, Clarke SA, Mills EG, Patel B, Modi M, Phylactou M, Eng PC, Thurston L, Alexander EC, et al. Thyroid function before, during, and after COVID-19. Journal of Clinical Endocrinology and Metabolism 2021106 e803-e811. (https://doi.org/10.1210/clinem/ dgaa830)

8 Campi I, Bulgarelli I, Dubini A, Perego GB, Tortorici E, Torlasco C, Torresani E, Rocco L, Persani L \& Fugazzola L. The spectrum of thyroid function tests during hospitalization for SARS COV-2 infection. European Journal of Endocrinology 2021184 699-709. (https://doi. org/10.1530/EJE-20-1391)

9 Giovanella L, Ruggeri RM, Petranović Ovčariček P, Campenni A, Treglia G \& Deandreis D. SARS-CoV-2-related thyroid disorders: a synopsis for nuclear medicine thyroidologists. European Journal of Nuclear Medicine and Molecular Imaging 202148 1719-1723. (https:// doi.org/10.1007/s00259-021-05316-0)

10 Caron P. Thyroid disorders and SARS-CoV-2 infection: from pathophysiological mechanism to patient management. Annales d'Endocrinologie 202081 507-510. (https://doi.org/10.1016/j. ando.2020.09.001)

11 Scappaticcio L, Pitoia F, Esposito K, Piccardo A \& Trimboli P. Impact of COVID-19 on the thyroid gland: an update. Reviews in Endocrine and Metabolic Disorders 2020 [epub]. (https://doi.org/10.1007/s11154-02009615-z)

12 Rotondi M, Coperchini F, Ricci G, Denegri M, Croce L, Ngnitejeu ST, Villani L, Magri F, Latrofa F \& Chiovato L. Detection of SARS-COV-2 receptor ACE-2 mRNA in thyroid cells: a clue for COVID-19-related subacute thyroiditis. Journal of Endocrinological Investigation $2021 \mathbf{4 4}$ 1085-1090. (https://doi.org/10.1007/s40618-020-01436-w)

13 Mithal A, Jevalikar G, Sharma R, Singh A, Farooqui KJ, Mahendru S, Krishnamurthy A, Dewan A \& Budhiraja S. High prevalence of diabetes and other comorbidities in hospitalized patients with COVID-19 in Delhi, India, and their association with outcomes. Diabetes and Metabolic Syndrome 202115 169-175. (https://doi.org/10.1016/j. dsx.2020.12.029)

14 Zhang Y, Lin F, Tu W, Zhang J, Choudhry AA, Ahmed O, Cheng J, Cui Y, Liu B, Dai M, et al. Thyroid dysfunction may be associated with poor outcomes in patients with COVID-19. Molecular and Cellular Endocrinology 2021521 111097. (https://doi.org/10.1016/j. mce.2020.111097)

15 Hariyanto TI \& Kurniawan A. Thyroid disease is associated with severe coronavirus disease 2019 (COVID-19) infection. Diabetes and Metabolic Syndrome 202014 1429-1430. (https://doi.org/10.1016/j. dsx.2020.07.044)

16 Gerwen M van, Alsen M, Little C, Barlow J, Naymagon L, Tremblay D, Sinclair CF \& Genden E. Outcomes of patients with

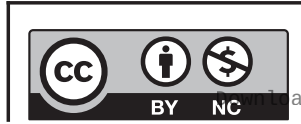
This work is licensed under a Creative Commons Attribution-NonCommercial 4.0 International License. ded from Bioscientifica.com at 04/26/2023 03:33:15AM 
hypothyroidism and COVID-19: a retrospective cohort study. Frontiers in Endocrinology 202011 565. (https://doi.org/10.3389/ fendo.2020.00565)

17 Brix TH, Hegedüs L, Hallas J \& Lund LC. Risk and course of SARSCoV-2 infection in patients treated for hypothyroidism and hyperthyroidism. Lancet: Diabetes and Endocrinology 20219 197-199. (https://doi.org/10.1016/S2213-8587(21)00028-0)

18 Jevalikar G, Mithal A, Singh A, Sharma R, Farooqui KJ, Mahendru S, Dewan A \& Budhiraja S. Lack of association of baseline

25-hydroxyvitamin D levels with disease severity and mortality in
Indian patients hospitalized for COVID-19. Scientific Reports 202111 6258. (https://doi.org/10.1038/s41598-021-85809-y)

19 Chaker L, Ligthart S, Korevaar TIM, Hofman A, Franco OH, Peeters RP $\&$ Dehghan A. Thyroid function and risk of type 2 diabetes: a population-based prospective cohort study. BMC Medicine 201614 150. (https://doi.org/10.1186/s12916-016-0693-4)

20 Lillevang-Johansen M, Abrahamsen B, Jørgensen HL, Brix TH \& Hegedüs L. Duration of over- and under-treatment of hypothyroidism is associated with increased cardiovascular risk. European Journal of Endocrinology 2019180 407-416. (https://doi.org/10.1530/EJE-19-0006)

Received in final form 22 September 2021

Accepted 18 October 2021

Accepted Manuscript published online 18 October 2021
This work is licensed under a Creative Commons Attribution-NonCommercial 4.0 International License. 